\title{
CORONAL LOOPS AND THEIR MODELING
}

\author{
G.H.J. VAN DEN OORD \\ Sterrekundig Instituut Utrecht \\ P.O. Box 80.000, 3508 TA Utrecht, The Netherlands \\ AND \\ F. ZUCCARELLO \\ Istituto di Astronomia dell'Università, Città Universitaria, \\ Viale A. Doria, I-95125 Catania, Italy
}

\begin{abstract}
We discuss the theory of quasi-static coronal loops, introducing a phase plane representation to study loop solutions independently of specific boundary conditions. Emphasis is put on the effects of loop expansion, heat input and gravitational stratification on the differential emission measure, and on the intrinsic limitations of spectroscopic observations for deriving loop parameters. We show that certain classes of published loop solutions cannot actually exist. For expanding loops new classes of loop solutions, with rather special properties, are presented. Special attention is paid to loops in binary systems and on rapidly rotating stars.
\end{abstract}

\section{Introduction}

Solar-like magnetic activity can be found in all stars possessing convective envelopes and rotating relatively rapidly. The study of individual coronal loops on stars is hampered by the lack of spatial resolution, but indirect means exist to derive some information. The observed decay of stellar flares permits estimates for the typical dimensions of the flaring loop (van den Oord and Mewe 1989). X-ray observations of eclipsing binary systems such as Algol (White et al. 1986, van den Oord and Mewe 1989, Stern et al. 1992), AR Lac (Walter et al. 1983, White et al. 1990, Siarkowski 1992), ER Vul (White et al. 1987) and TY Pix (Preś et al. 1995) indicate that hot stellar-sized coronal loops exist in these systems, next to more compact solar-like active regions. This is substantiated by VLBI observations of RS 
CVn systems (Lestrade et al. 1984, Mutel et al. 1984) and Algol (Clark et al. 1975, 1976) which indicate the presence of gyro-synchrotron emission from halo-like structures for which the upper limit of the source size is comparable to the overall size of the binary.

The most direct means for obtaining information about the source structure is by applying a differential emission measure (DEM) analysis to an observed spectrum. The next step is then to interpret the recovered DEM in terms of coronal loop models. Due to lack of sufficient spectral resolution in the EUV/X-ray range it was until recently not possible to derive DEMs with sufficient resolution in temperature. The only exception are the analyses of the spectra of Capella and $\sigma^{2} \mathrm{CrB}$ by Schrijver et al. (1989) using the EXOSAT transmission grating spectrometer. These authors were able to show that the observed DEMs cannot be reconciled with the 'standard' loop models of Rosner et al. (1978, hereafter RTV) but require expanding loop geometries as have been discussed by Vesecky et al. (1979, hereafter VAU).

Recently, the EUVE has produced spectra which do permit a DEM analysis and in the near future this will be possible with $A X A F$ and XMM. An issue which will become increasingly important concerns the relation between coronal loop models and the actually 'observed' DEM, in other words: how well does the DEM distinguish between different coronal loop models? The extensive literature on coronal loops cannot be adequately summarized in this brief review but is extensively reviewed in the book by Bray et al. (1991). Even for simple loop models one often has to rely on numerical techniques. This makes it difficult to obtain physical insight into why a solution shows a specific behaviour. We therefore use in this paper a so-called diagnostic diagram as well as a phase plane representation to discuss and predict the behaviour of loop solutions. We restrict ourselves to quasi-static loops because these are simple to model and permit a comparison with observations. Dynamical models, involving plasma flows, require a spectral resolution at X-ray wavelengths which cannot be achieved with the present instrumentation. A more extensive discussion of previously published loop solutions will be given in van den Oord and Zuccarello (1996, hereafter VDOZ).

The contents of this review is as follows. In Sect. 2 we discuss the basic equations, in Sect. 3 constant pressure loops, in Sect. 4 loops in hydrostatic equilibrium, in Sect. 5 the effect of loop expansion and in Sect. 6 the effect of gravity reversals in loops. Our conclusions are presented in Sect. 7. 


\section{Basic equations}

The thermodynamical structure of a loop in quasi-static equilibrium is determined by the equation for conservation of energy

$$
\frac{d F_{\mathrm{c}}(s)}{d s}=A(s)\left(E_{\mathrm{heat}}(s)-E_{\mathrm{rad}}(s)\right)
$$

the equation for hydrostatic equilibrium

$$
\frac{d p(s)}{d s}=m_{\mathrm{H}} n(s) g(s)
$$

and an equation of state. In these expressions $s$ is the coordinate along the magnetic field, $A(s)$ is the cross-section of the loop , $p(s)$ is the pressure, $n(s)$ the electron density, $m_{\mathrm{H}}$ is the hydrogen mass, and $g(s)$ is the gravitational acceleration. The classical conductive flux through the cross-section of a loop is given by $F_{\mathrm{c}}(s)=-A(s) \kappa_{0} T^{5 / 2}(d T / d s)$ with $\kappa_{0}=8.810^{-7} \mathrm{erg} \mathrm{cm}^{-1} \mathrm{~s}^{-1} \mathrm{~K}^{-7 / 2}$ and $T$ the temperature. The optically thin radiative losses are given by $E_{\mathrm{rad}}(s)=n^{2} \Psi(T) \quad\left(\mathrm{erg} \mathrm{cm}^{-3} \mathrm{~s}^{-1}\right)$, with $\Psi(T)$ the emissivity, and the heat input is given by $E_{\text {heat }}$. Eq. (1) shows that the difference between heating and radiative losses at a given position in the loop is balanced by the conductive energy. The equations given above require three 'external' functions to be specified. These are the variation of the cross-section of the loop $A(s)$, the gravitational acceleration along the loop $g(s)$ and the heat input in the loop $E_{\text {heat }}(s)$. We assume the presence of a fully ionized hydrogen plasma, except for the emissivity $\Psi(T)$, taken from van den Oord and Barstow (1988), which includes both line and continuum emission from a plasma with solar abundances. Above 0.1 MK a useful approximation is $\Psi(T)=10^{-18.8} / \sqrt{T}$.

By using the equation of state for a hydrogen plasma $(p=2 n k T)$ Eqs. (1) and (2) can be written as a system of coupled nonlinear ordinary differential equations

$$
\begin{aligned}
\frac{d T}{d s} & =-\frac{F_{\mathrm{c}}}{A \kappa_{0} T^{5 / 2}} \\
\frac{d F_{\mathrm{c}}}{d s} & =A p^{2}\left(\frac{E_{\mathrm{heat}}}{p^{2}}-\frac{\Psi(T)}{4 k^{2} T^{2}}\right), \\
\frac{d p}{d s} & =m_{\mathrm{H}} \frac{p}{2 k T} g .
\end{aligned}
$$

These equations can be integrated given the three boundary conditions at the base of the loop for the temperature $\left(T=T_{\mathrm{b}}\right)$, the conductive flux $\left(F_{\mathrm{c}}=F_{\mathrm{c}, \mathrm{b}}\right)$ and the pressure $\left(p=p_{\mathrm{b}}\right)$. 
The differential emission measure (DEM) is the weighting function for the contribution of the plasma at each temperature to the observed spectrum. If $g(\lambda)$ is the observed spectrum and $f(\lambda, T)$ is the emissivity of the plasma at temperature $T$ then

$$
\begin{aligned}
g(\lambda) & =\int f(\lambda, T) n^{2}(T) d V(T)=\int f(\lambda, T) n^{2}(T) A(T)\left|\frac{d T}{d s}\right|^{-1} d T \\
& \equiv \int f(\lambda, T) \operatorname{DEM}(T) d \log T
\end{aligned}
$$

Using the expression for the conductive flux to eliminate $d T / d s$ gives

$$
\operatorname{DEM}(T) \equiv \frac{\kappa_{0} \ln 10}{4 k^{2}} \frac{A^{2} p^{2} T^{3 / 2}}{\left|F_{\mathrm{c}}\right|} \mathrm{cm}^{-3}
$$

where $A, p$ and $F_{\mathrm{c}}$ depend (implicitly) on temperature $T$. One faces basically two problems when working with a DEM: 1) it depends in a nontrivial way on the loop parameters, and 2) the DEM must be recovered from a Fredholm equation of the first kind (Eq. (6)) which has no unique solution. Loops with different (thermodynamical) structures can result in almost identical DEMs. Ideally one would like to know the DEM with an as good temperature resolution as possible. Apart from the problems which noise causes in recovering a DEM from an observed spectrum, there is an intrinsic limit to the temperature resolution which can be achieved. Spectral lines provide the most accurate temperature diagnostics but lines form over a finite temperature range, typically a factor two in temperature or $\Delta \log T=0.3$, determined by the ionization balance and the excitation function. When a spectrum contains sufficient lines at slightly different formation temperatures one may achieve a resolution of $\Delta \log T=0.1$. This intrinsic limit to the temperature resolution at which a DEM can be recovered is independent of the spectral resolution of the instrument.

\section{Constant pressure loops}

The simplest loop models are those for which the pressure is taken constant implying that $g=0$ in Eq. (5) and that the pressure scale height is infinite. This leaves Eqs. (3) and (4) to be solved. These equations form a system of coupled nonlinear differential equations. When $E_{\text {heat }}$ and $A$ are functions of the loop coordinate $s$ the system is called non-autonomous because the right-hand side depends explicitly on $s$. Such systems have to be solved numerically. Let us for the moment assume the $E_{\text {heat }}$ and $A$ are constant so that the system becomes autonomous. The appropriate way to study the properties of such a system is by investigating how the solutions run in 

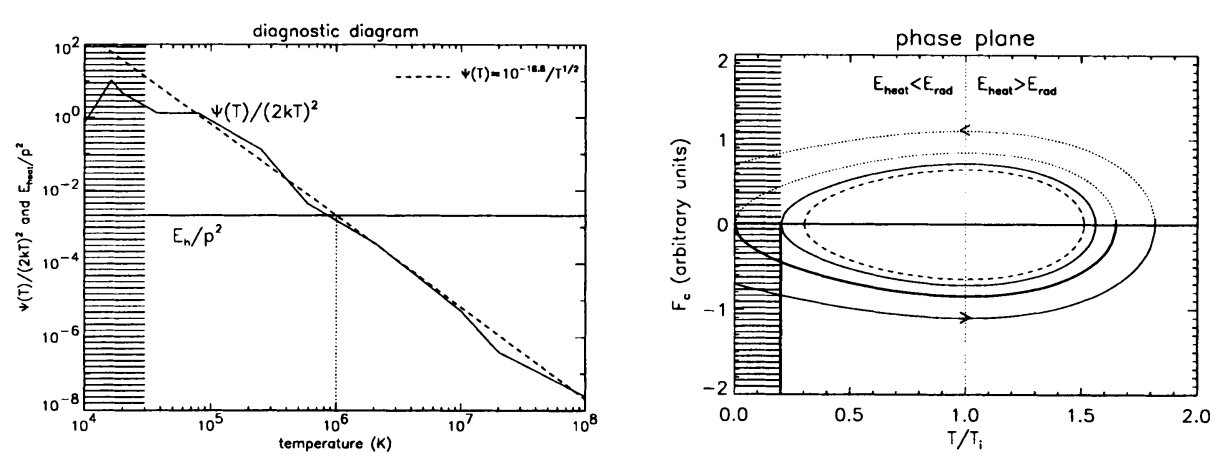

Figure 1. (a) Diagnostic diagram showing the two terms which determine the run of $d F_{\mathrm{c}} / d s: E_{\text {heat }} / p^{2}$ and $\Psi(T) /(2 k T)^{2}$. The dashed line is for the approximation $\Psi(T)=10^{-18.8} T^{-0.5}$. The thick solid line indicates $E_{\text {heat }} / p^{2}$, in this case taken constant. (b) Corresponding phase plane $\left(T, F_{\mathrm{c}}\right)$ showing some phase space trajectories of the solutions. Physically meaningful solutions start at the thick vertical line.

the $\left(T, F_{\mathrm{c}}\right)$ phase plane. Essential ingredients are the critical points, that is, those values of $T$ and $F_{\mathrm{c}}$ for which $d T / d s=d F_{\mathrm{c}} / d s=0$. With the above assumptions the critical point, labeled with index ' $\mathrm{i}$ ', is given by $F_{\mathrm{c}, \mathrm{i}}=0$ and by temperature $T_{\mathrm{i}}$ which is the solution of $E_{\text {heat }} / p^{2}=\Psi\left(T_{\mathrm{i}}\right) /\left(4 k^{2} T_{\mathrm{i}}^{2}\right)$. In Fig. 1a we show a diagnostic diagram depicting the functions $E_{\text {heat }} / p^{2}$ and $\Psi\left(T_{\mathrm{i}}\right) /\left(4 k^{2} T^{2}\right)$ which determine the sign of Eq. (4). Because the curves intersect only once, here at $T_{\mathrm{i}}=10^{6} \mathrm{~K}$, there is only one critical point. Linearizing the equations around the critical point shows that this point is a centre point so that all solutions encircle this point (anti-clockwise). In fact, for an autonomous system it is often possible to construct a first integral by dividing Eqs. (3) and (4) and integrating $d F_{\mathrm{c}} / d T$. The first integral gives the phase space trajectories shown in Fig. 1b. Physically meaningful solutions are determined by choosing appropriate boundary conditions and thereby selecting a specific phase space trajectory. There are two important constraints for the choice of boundary conditions: 1) the coronal loop solution must be able to connect continuously to the chromospheric part of the loop and 2) the optical thin approximation for the radiative losses must remain valid. Below a temperature of approximately $310^{4} \mathrm{~K}$ radiative transfer effects become increasingly important but, on the other hand, this temperature is at a chromospheric value so that a choice $T_{\mathrm{b}}=310^{4} \mathrm{~K}$ guarantees that both constraints are satisfied. The choice for $F_{\mathrm{c}, \mathrm{b}}$ depends on whether the chromosphere is (partly) energetically fed by the conductive flux out of the coronal volume, a matter still unresolved. Somewhere within the coronal loop the temperature has a maximum, for hot coronal loops, or a local minimum, for loops with a temperature inversion, so that at the corresponding temperature, labeled ' $\mathrm{a}$ ', $d T / d s=0$ and consequently 
$F_{\mathrm{c}}\left(T_{\mathrm{a}}\right)=0$. We will refer to this point as the apex of the loop, although it does not necessarily have to correspond with the geometrical apex. The fact that the optically thin approximation breaks down below $310^{4} \mathrm{~K}$ is indicated by the hatched areas in Fig. 1.

Typical coronal loop solutions (see Fig. 1b) start at the right-hand boundary of the hatched area, then show an increase of the temperature while the conductive flux becomes increasingly negative. At $T=T_{\mathrm{i}}$ the conductive flux reaches a minimum and then becomes rapidly zero at $T=T_{\mathrm{a}}$ which corresponds to the apex temperature. In principle one can continue to follow the solutions in the upper part of the phase plane and look for loops with a temperature inversion at their apex (a second intersection with the line $F_{\mathrm{c}}=0$ ). Fig. $1 \mathrm{~b}$ shows however that all solutions which start out at a chromospheric temperature do not reach $F_{\mathrm{c}}=0$ at temperatures below $T_{\mathrm{i}}$, or do so only in the 'forbidden' hatched region. This implies that constant pressure loops cannot have cool apexes. The dashed solution in Fig. $1 \mathrm{~b}$ is unphysical because it cannot be connected to the chromosphere. In the past various authors have taken the base temperature $T_{\mathrm{b}}$ at higher values $\left(\geq 10^{5} \mathrm{~K}\right)$ in order to avoid the problems with the radiative loss curve at low temperatures. This approach may result in studying solutions which are disconnected from the chromosphere. The solution on which RTV based their scaling laws corresponds to the boundary conditions $\left(T_{\mathrm{b}}=0, F_{\mathrm{c}, \mathrm{b}}=0\right)$ (thick line in Fig. 1b).

Another interesting and very important aspect is that the difference between $T_{\mathrm{i}}$ and $T_{\mathrm{a}}$ is small, in fact below a factor two, even for loops with a large conductive flux at the base. For RTV loops one has $T_{\mathrm{a}}=$ $3.5^{0.4} T_{\mathrm{i}}=1.65 T_{\mathrm{i}}$ (see Fig. 1b). Below temperature $T_{\mathrm{i}}$ the structure of a loop is determined by the balance between conduction and radiation (giving DEM $\sim T^{3 / 2}$ ) and the DEM contains no information on the heating. At temperatures $T_{\mathrm{i}}<T \leq T_{\mathrm{a}}$, corresponding geometrically to the largest part of a loop, conduction balances heating and, to a lesser extent, radiation. This part of the loop, from which in principle something could be learned about the heating process, is however mapped into one, or at most a few, DEM bin(s) of width $\Delta \log T=0.1$. In fact, stronger heating at $T>T_{\mathrm{i}}$ results in $T_{\mathrm{a}} / T_{\mathrm{i}}$ approaching unity, making the situation worse. This implies that from spectroscopic studies little can be learned about coronal heating, within the context of coronal loop models. The insensitivity of the solutions to the heating at $T<T_{\mathrm{i}}$ and the reduction of $T_{\mathrm{a}} / T_{\mathrm{i}}$ for increased heating at $T>T_{\mathrm{i}}$ is demonstrated in Fig. 2 .

Clearly, the temperature $T_{\mathrm{i}}$ at which the heating and the radiative losses balance plays an important role whereas below this temperature heating is not important. This was already noted by Craig et al. (1978) which led these authors to consider so-called 'esoteric' heating functions which fol- 
diognostic diogrom for $T_{i}=10^{6} \mathrm{~K}$

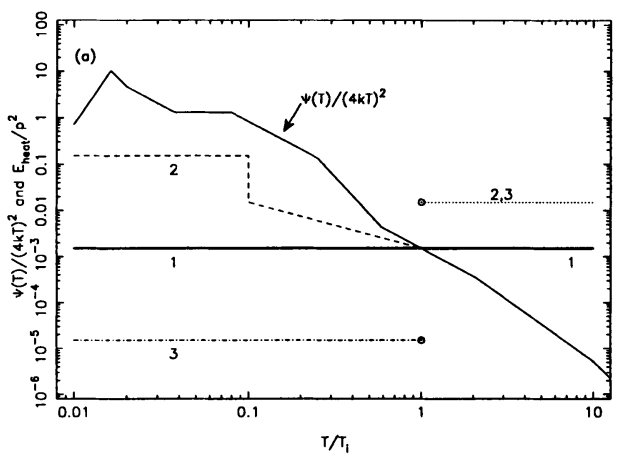

phose plane for $\mathrm{T}_{1}=10^{6} \mathrm{~K}$

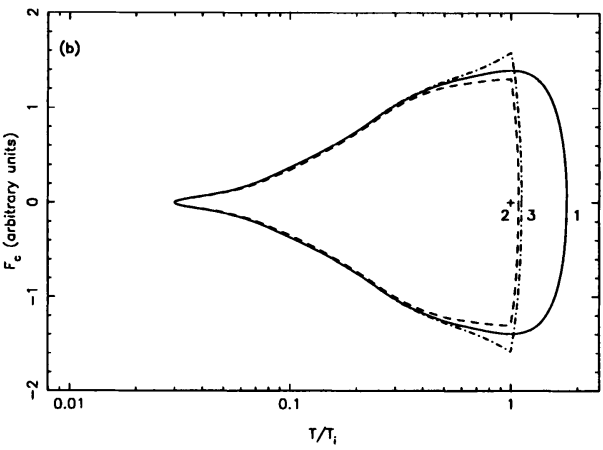

Figure 2. (a) Diagnostic diagram showing $\Psi(T) /(2 k T)^{2}$ and three $E_{\text {heat }} / p^{2}$ curves with one corresponding to constant heating ( 1 ; thick line). Also indicated are two heating functions with increased heating (2;dashed line) and reduced heating (3;dashed-dotted line) for $T<T_{\mathrm{i}}$. At $T=T_{\mathrm{i}}$ these heat functions intersect the $\Psi(T) /(2 k T)^{2}$ curve, the dashed-dotted line being discontinuous. At $T>T_{\mathrm{i}}$ these heat functions exceed the constant heat input by a factor ten (dotted line). (b) Phase plane trajectories for constant heat function $\left(1 ;\right.$ solid), increased heating below $T_{\mathrm{i}}(2 ;$ dashed $)$ and reduced heating below $T_{\mathrm{i}}$ (3;dashed-dotted). The non-constant heating solutions have increased heating above $T_{\mathrm{i}}$. Note that below $T_{\mathrm{i}}$ the solutions are almost independent of the heat input and that increased heating above $T_{\mathrm{i}}$ makes $T_{\mathrm{a}} \rightarrow T_{\mathrm{i}}$.
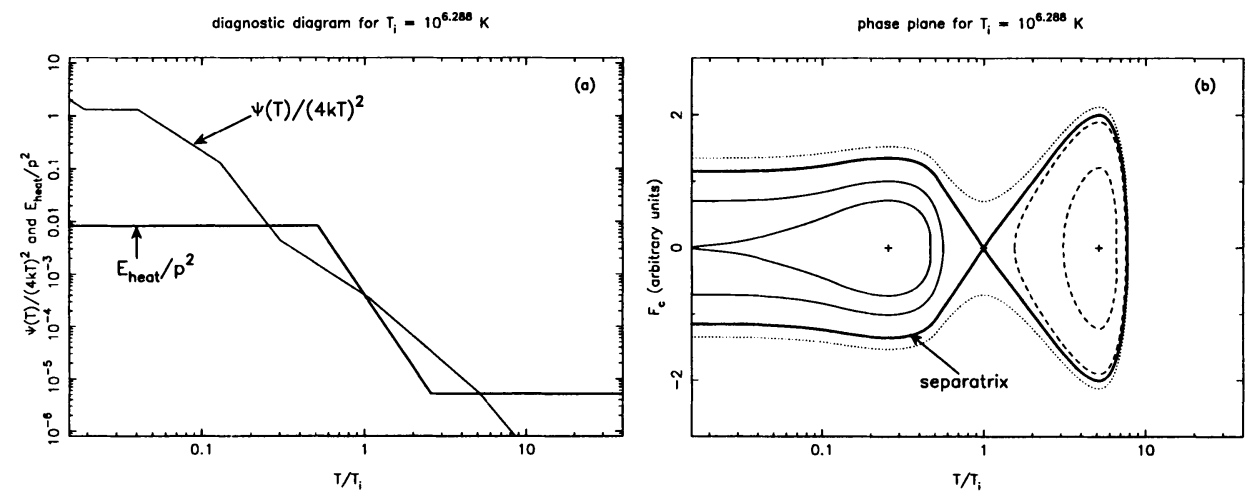

Figure 3. (a) Diagnostic diagram for a heating function that intersects the $\Psi(T) /(2 k T)^{2}$ curve three times. The central intersection is at $T=T_{\mathrm{i}}=10^{6.288} \mathrm{~K}$. (b) Phase plane containing two centre and one saddle point. The thick line indicates the separatrix. The dotted line indicates a solution outside the separatrix which can never result in a vanishing conductive flux at the base. The dashed solutions near the high-temperature centre point cannot connect to the chromosphere.

low the radiative loss curve over a large temperature range and intersect it several times. The fact that then alternating heating and radiative losses dominate led this authors to speculate that such loops would be characterized by temperature oscillations along the loop. This is however not the 
case. In Fig. 3a we show a heating function which crosses the radiative loss curve three times corresponding to the presence of three critical points. The corresponding phase plane of the solutions is given in Fig. 3b. The figure shows that the central intersection corresponds to a saddle point while the other intersections correspond to centre points. A local analysis (see VDOZ) shows that if near the critical point the radiative losses vary as $\Psi \sim T^{-\gamma}$ and the heating as $E_{\text {heat }} \sim T^{\delta}$, the critical point is a saddle point for $\delta+\gamma+2<0$. The interesting point is that the solutions can never cross the separatrix (thick line). The solutions encircling the high-temperature centre point are physically disconnected from the chromosphere. Only loops with a large conductive flux at the base can have high apex temperatures but there exists a region of non-accessible apex temperatures. The presence of the saddle point implies that within a loop the heating function cannot intersect the radiative loss curve more than once so that 'esoteric' heating functions do not appear in nature. Furthermore, any coronal heating mechanism must deposit energy near the loop apex, while switching off the heating below $T=T_{\mathrm{i}}$ does not affect the loop structure.

\subsection{SCALING LAWS}

Over the last decades scaling laws have received considerable attention because they are extremely useful when interpreting observations without spatial resolution. The exact form of any scaling law depends of course on the approximation for the radiative loss curve used (see e.g. Kuin and Martens 1982). One scaling law is related to the critical point in the phase plane $\left(E_{\text {heat }} / p^{2}=\Psi\left(7_{\mathrm{i}}\right) /\left(2 k T_{\mathrm{i}}\right)^{2}\right)$ while a second scaling law follows from integrating Eq. (3) over the (half) length of the loop. Because of the complexity of the integral authors normally assume $T_{\mathrm{b}} \downarrow 0$ and $F_{\mathrm{c}, \mathrm{b}} \downarrow 0$. The apex temperature and the loop length depend of course on the phase space trajectory one selects in Fig. 1b. In Fig. 4 we show the relation between $p, L$ and $T_{\mathrm{a}}$ when $T_{\mathrm{b}}=310^{4} \mathrm{~K}$ and $E_{\text {heat }}=10^{-3} \mathrm{erg} \mathrm{cm}^{-3} \mathrm{~s}^{-1}$, and for various values of the base conductive flux. The base conductive flux is expressed in the unit $F_{\text {limit }}$ which corresponds to $F_{\mathrm{c}}\left(T=310^{4} \mathrm{~K}\right)$ on the RTV trajectory. The vertical line indicates the pressure at which $T_{\mathrm{b}}=T_{\mathrm{i}}$ below which pressure no solutions exist. The figure shows that the relation between $p, L$ and $T_{\mathrm{a}}$ is fairly insensitive to the value of $F_{\mathrm{c}, \mathrm{b}}$. Diagrams like Fig. 4 are also useful to study the effect of varying $p$ (with $E_{\text {heat }}$ and $L$ constant) or varying $L$ (with $E_{\text {heat }}$ and $p$ constant). A discussion can be found in VDOZ. These type of variations were considered by Hood and Priest (1979) to show that for certain variations no adjacent (hot) equilibrium can be found. This would then result in catastrophic cooling due to a jump from equilibria with hot apexes towards equilibria with temperature 
inversions (cool apexes). Later it was shown by Hood and Anzer (1988) that the inverted solutions found by Hood and Priest are an artefact of the high base temperature $\left(T_{\mathrm{b}}=10^{6} \mathrm{~K}\right)$ employed by Hood and Priest. In other words, the boundary conditions at the base assumed by Hood and Priest do not permit a connection of the coronal part of the loop to the chromosphere. Given the fact that cool solutions do not exist, Fig. 4 indicates that for certain variations no equilibrium exists and the quasi-static assumption must break down. E.g., if $L$ and $E_{\text {heat }}$ are kept constant, an increase of the pressure results in a decrease of $F_{\mathrm{c}}$ until no further equilibria are found.
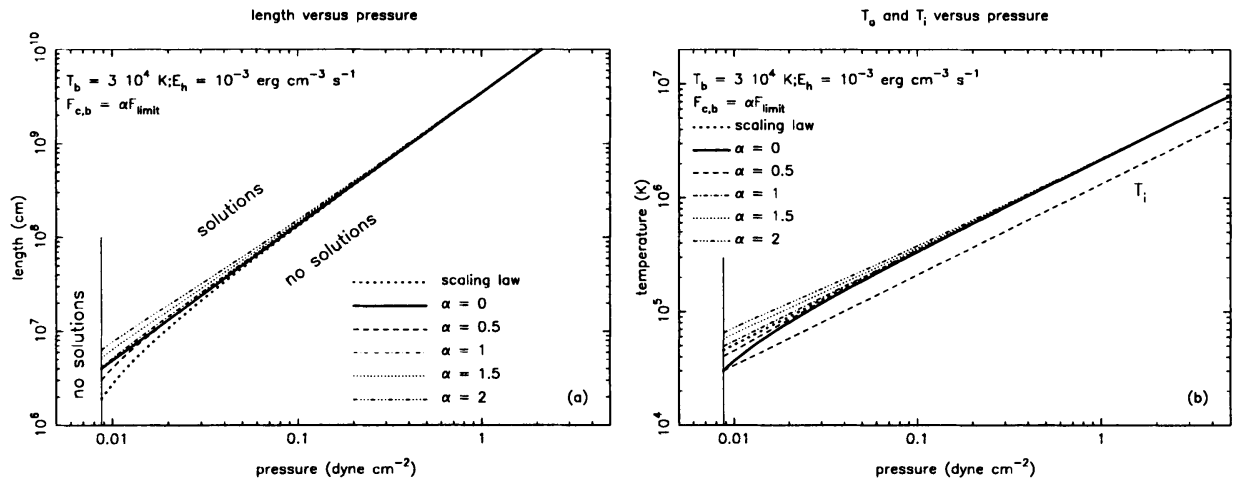

Figure 4. Resulting loop lengths (a) and apex temperatures (b) for the case of heating per unit volume $\left(E_{\text {heat }}=10^{-3} \mathrm{erg} \mathrm{cm}^{-3} \mathrm{~s}^{-1}\right)$ and radiative losses according to $\Psi(T)=\Psi_{0} / \sqrt{T}$. In this case $T_{\mathrm{i}}=\left(p^{2} \Psi_{0} /\left(4 k^{2} E_{\text {heat }}\right)\right)^{2.5}$. The vertical lines indicate the pressure for which $T_{\mathrm{i}}=T_{\mathrm{b}}$. The loop lengths and apex temperatures are determined for various values of the base conductive flux $F_{\mathrm{c}, \mathrm{b}}=\alpha F_{\text {limit }}$ with $F_{\text {limit }}$ the conductive flux at $T_{\mathrm{b}}=310^{4} \mathrm{~K}$ on the RTV trajectory which runs through $\left(T, F_{\mathrm{c}}\right)=(0,0)$. Also indicated is the scaling law (dots) of RTV. In (b) the lower dashed line indicates $T_{\mathrm{i}}$ as a function of pressure.

We note that analytic scaling laws can only be found for autonomous systems, that is, systems for which a first integral exists. Apart from the case of constant heating and constant cross-section, this integral also exists for scalings like $A \sim T^{\alpha}$ and $E_{\text {heat }} \sim T^{\beta}$ (see e.g. Landini and MonsignoriFossi, 1981). A scaling like $A \sim T^{\alpha}$ implies unrealistically large expansions of loops given the typical values for $T_{\mathrm{b}}$ and $T_{\mathrm{a}}$. A scaling like $E_{\mathrm{heat}} \sim T^{\beta}$ does not strongly influence the loop structure because below $T_{\mathrm{i}}$ the heating is not important and above $T_{\mathrm{i}}$ it will only reduce the apex temperature compared to constant heating: $T_{\mathrm{a}} / T_{\mathrm{i}}=(3.5+\beta)^{1 /(2.5+\beta)}$.

\section{Loops in hydrostatic equilibrium}

The effect of hydrostatic equilibrium $(g \neq 0)$ on the structure of coronal loops is small. This can be easily seen from Fig. 1a. When the pressure 
drops along a loop the $E_{\text {heat }} / p^{2}$-curve turns slightly upward with increasing temperature, so that $T_{\mathrm{i}}$ slightly shifts to lower temperatures. Because the difference between the $E_{\text {heat }} / p^{2}$ and the $\Psi(T) /(2 k T)^{2}$ curves then increases above $T_{\mathrm{i}}$, the conductive flux becomes more rapidly zero, so that also the apex temperature is lowered. Because of the large coronal pressure scale height $\left(310^{10} T_{7} \mathrm{~cm}\right)$ these effects are only small. Because DEM $\sim p^{2}$ the influence of hydrostatic equilibrium is to make the DEM-distribution a bit flatter at the high temperature end (see VDOZ).

phase diagram

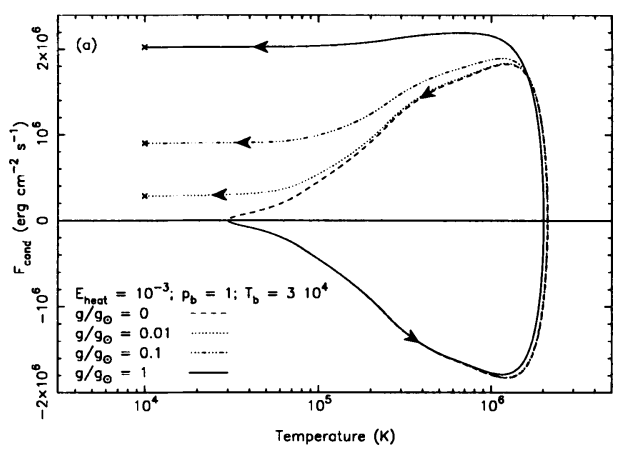

diagnostic diagram

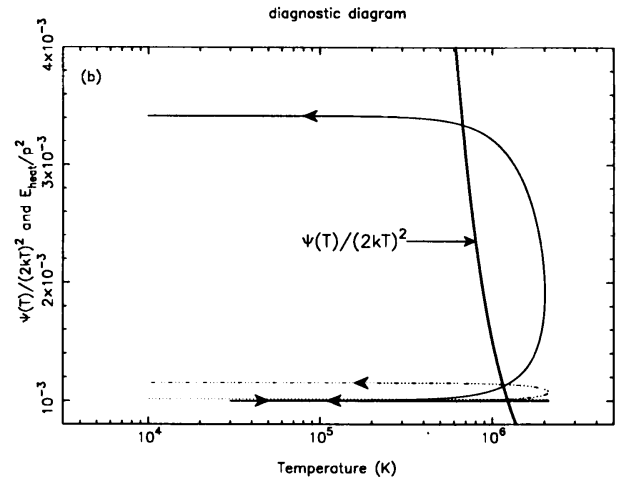

Figure 5. (a) Phase plane diagram for solutions resulting from integration from the base $\left(T_{\mathrm{b}}=310^{4} \mathrm{~K}, p_{\mathrm{b}}=1\right.$ dyne $\left.\mathrm{cm}^{2}, F_{\mathrm{c}, \mathrm{b}}=0\right)$ to the apex for various values of the gravitational acceleration. The arrows point towards the apex. For $g=0$ a closed trajectory is found with $T_{\mathrm{b}}=T_{\mathrm{a}}$. A finite gravitational acceleration results in a shift of the apex temperature to values below the base temperature. For solutions with $g / g_{\odot}>10^{-3}$ no apex temperatures can be found with realistic values, say $T_{\mathrm{a}} \geq 10^{4}$. For those solutions the calculations where terminated at $10^{4} \mathrm{~K}$ (crosses). (b) Corresponding diagnostic diagram for the solutions in panel (a). Due to the decrease of the pressure towards the apex the $E_{\text {heat }} / p^{2}$ curve has now a lower and an upper branch which (almost) coincide when $g$ is zero (or small).

Several authors (Serio et al. 1981, Collier Cameron 1988) have considered the possibility that loops with a temperature inversion might exist under hydrostatic equilibrium. These loops have a local temperature minimum at/near their apex. The interpretation of the results published in the literature is hampered by the fact that the various authors do not present the phase plane trajectories of their solutions. A loop with a temperature inversion corresponds to a full encircling of the critical point in the phase plane until $F_{\mathrm{c}}\left(T_{\mathrm{a}}\right)=0$ for $T_{\mathrm{a}}<T_{\mathrm{i}}$ : one intersection with the line $F_{\mathrm{c}}=0$ corresponds to the point where the temperature reaches a maximum while the next intersection occurs at the inverted apex temperature. In Fig. 5 we show what happens when the gravity is increased in a loop. When $g=0$ (constant pressure loops) the phase trajectory is a closed curve starting and ending at $T_{\mathrm{b}}=310^{4} \mathrm{~K}$ and $F_{\mathrm{c}, \mathrm{b}}=0$. If however $g$ is increased, the 
point associated with the cool apex moves rapidly into the region where the coronal approximation breaks down. For $g=g_{\odot}$ we even stopped the computation because the apex temperature becomes almost zero. We conclude that inverted loop solutions do not exist for realistic values of the gravitational acceleration. The apex temperature lies necessarily at such low values that the radiative losses cannot be appropriately described by the optically thin radiative loss curve. In fact, it can be shown that if one integrates from a cool apex (e.g. $T_{\mathrm{a}}=310^{4} \mathrm{~K}$ ) towards the foot points, one cannot find solutions which at the base connect to the chromosphere (see VDOZ).

\section{Expanding loops}

The thermodynamical structure of expanding loops has been numerically investigated by VAU and Collier Cameron (1988). In order to compare the solutions for a varying cross-section with those of a constant cross-section it is useful to redefine the conductive flux. In Eq. (3) the conductive flux $F_{\mathrm{c}}(s)$ is defined as the flux through the whole cross-section of the loop at position $s: F_{\mathrm{c}}(s)=-A(s) \kappa_{0} T^{5 / 2}(d T / d s) \mathrm{erg} \mathrm{s}^{-1}$. Let us now define the flux per unit cross-section: $G_{\mathrm{c}}(s) \equiv F_{\mathrm{c}}(s) / A(s)$. Eqs. (3) and (4) can then be written as

$$
\begin{gathered}
\frac{d T}{d s}=-\frac{G_{\mathrm{c}}}{\kappa_{0} T^{5 / 2}} \\
\frac{d G_{\mathrm{c}}}{d s}=E_{\text {heat }}-\frac{p^{2}}{4 k^{2}} \frac{\psi(T)}{T^{2}}-G_{\mathrm{c}} \frac{d \ln A}{d s}
\end{gathered}
$$

These equations have the same form as Eqs. (3) and (4) except for the last term in Eq. (9) which accounts for the effects of expansion. Because in normal loops $G_{\mathrm{c}} \leq 0$ the term $-G_{\mathrm{c}}(d \ln A / d s)$ can be considered as an extra heat source in the system, a point already noted by VAU. From our previous discussion we can directly describe the effect of such an increased heating: for constant boundary conditions it lowers the temperature $T_{\mathrm{a}}$. Note however that $G_{\mathrm{c}}=0$ at the apex and, depending on the boundary conditions, also at the base. At $T=T_{\mathrm{i}},\left|G_{\mathrm{c}}\right|$ has a maximum but decreases rapidly towards $T_{\mathrm{a}}$. In a diagnostic diagram like Fig. 1a the effective 'heating' function $\left(E_{\text {heat }}-G_{\mathrm{c}} d \ln A / d s\right) / p^{2}$ will first turn upward for $T<T_{\mathrm{i}}$ and then turn downward to approach $E_{\text {heat }} / p^{2}$ at $T_{\mathrm{a}}$. The effect of loop expansion is most noticeable in the DEM-distribution given the DEM $\sim A^{2} / F_{\mathrm{c}}=A / G_{\mathrm{c}}$ dependence which implies a steepening of the DEM. Because a DEM reflects mostly the ratio of lines formed at different temperatures, the observed ratio of 'hot' and 'cool' lines can be mimicked by allowing a loop expansion.

A special class of solutions exist for exponentially expanding loops: $A(s)=A_{\mathrm{b}} \exp \left(s / H_{1}\right)$ with $H_{1}$ the scale height for the expansion of the 
loop. In that case the system becomes autonomous and a local analysis can be made near the critical point. An important parameter is $R \equiv$ $4(\gamma+2) H_{1}^{2} E_{\text {heat }} /\left(\kappa_{0} T_{\mathrm{i}}^{3.5}\right)$ (for $\left.\Psi \sim T^{-\gamma}\right)$. For $R>1$ the critical point is a spiral point and for $R<1$ a stable node point. The typical trajectories for these cases are shown in Fig. 6. These solutions are first identified in VDOZ where a further discussion of the critical point analysis can be found. The solutions have several interesting properties: 1) $T_{\mathrm{a}}=T_{\mathrm{i}}$ so that for high values of $E_{\text {heat }} / p^{2}$ cool apexes can be found; 2) all solutions with $R>1$ have temperature inversions and for $R<1$ temperature inversions are found for a finite conductive flux at the base; 3 ) because the phase space trajectories cross certain temperature ranges multiple times, the DEM will show a strong 'shoulder' starting near $T_{\mathrm{a}}$. These solutions are especially interesting because they permit the formation/presence of cool filament-like plasma near the apex without the need of a radiative instability. We note that exponentially expanding flux tubes occur naturally in linear force-free arcades so that arcade-like structures may have a complicated thermodynamical structure with strong X-ray emission coming from the apex or with cool material near the apex, depending on the value of $E_{\text {heat }} / p^{2}$.
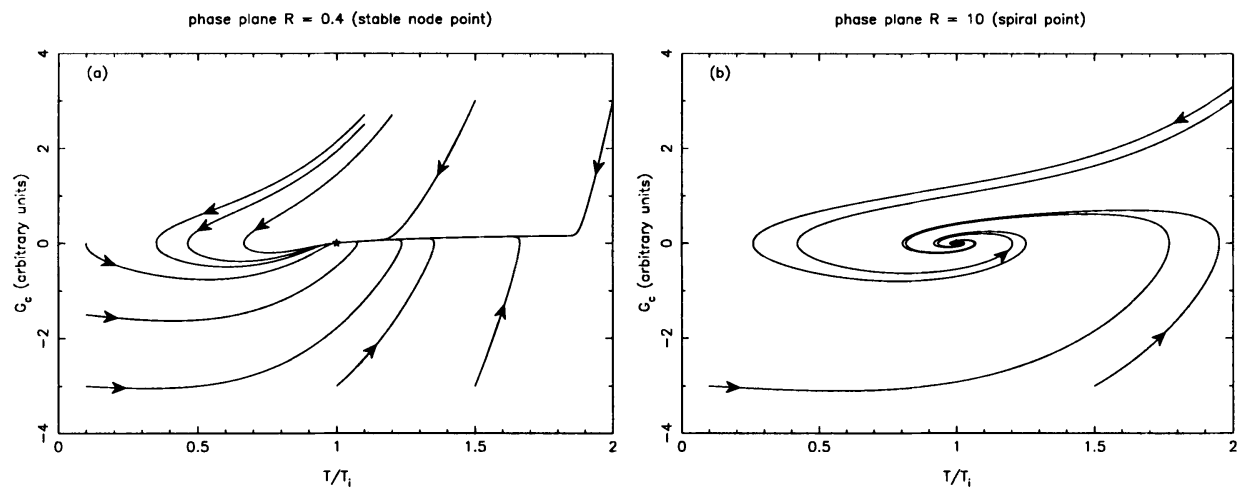

Figure 6. Phase space trajectories for an exponentially expanding loop. For these solutions $T_{\mathrm{a}}=T_{\mathrm{i}}$ and the critical point (asterisk), for which the heating balances the radiative losses, is an attractor. The classification of the critical point depends on parameter $R$ (see text). When $R<1$ the critical point is a stable node point (a) and when $R>1$ a spiral point (b).

\section{Reversed gravity potentials}

A special situation exists when the gravitational acceleration changes sign somewhere along a loop. Such a situation exists for loops on fast rotating stars (see Collier Cameron 1988) and for loops in binary systems which experience the gravitational pull by the binary companion (see VDOZ). In 
phose diogram
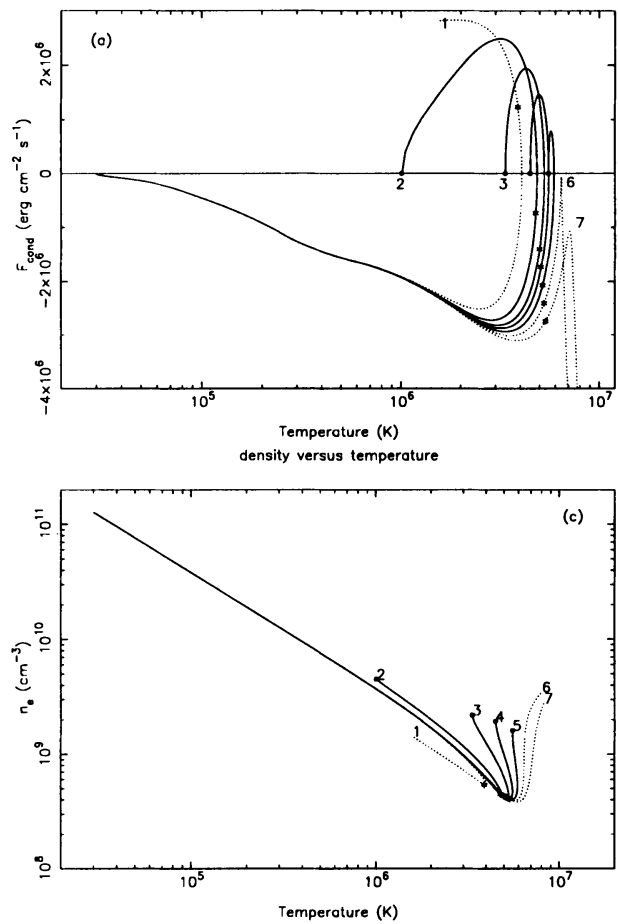

diognostic diagram
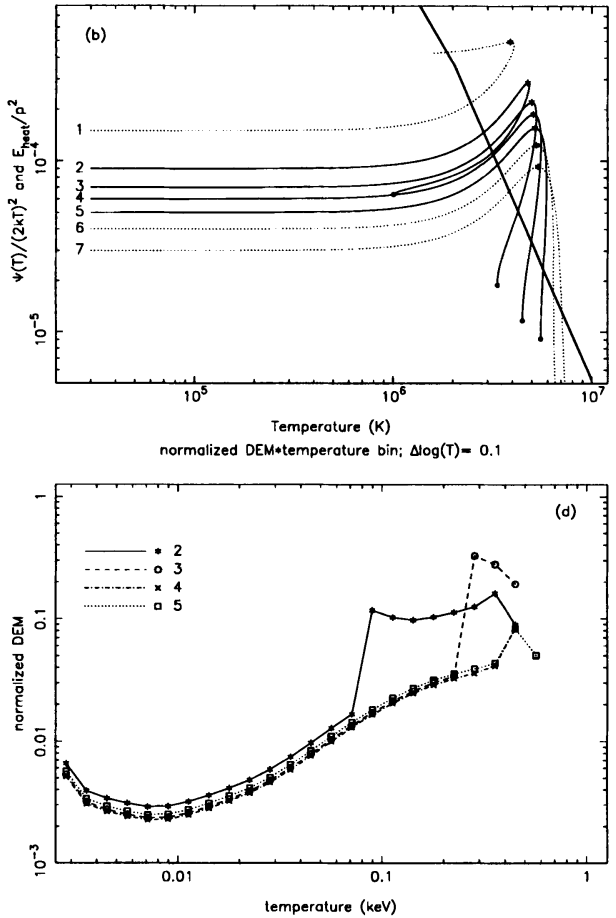

Figure 7. Solutions for coronal loops on a rapidly rotating star with $M=M_{\odot}, R=R_{\odot}$ and the rotation period chosen in such a way that the co-rotation radius is located at $0.5 R_{\odot}$ above the stellar surface. The boundary conditions at the base of the loop are $T_{\mathrm{b}}=310^{4} \mathrm{~K}, p_{\mathrm{b}}=1$ dyne $\mathrm{cm}^{-2}$ and $F_{\mathrm{c}, \mathrm{b}}=0$. The curves labeled (1) - (7) correspond to heating rates of $15,10,9,8,7,6,4 \times 10^{-5} \mathrm{erg} \mathrm{cm}^{-3} \mathrm{~s}^{-1}$ respectively. (a) Phase space trajectories. The crosses indicate the position of the co-rotation radius. The dots indicate the apexes of loops with a temperature inversion. The co-rotation radius is found at increasingly lower increasingly lower values of $F_{\mathrm{c}}$. (b) Diagnostic diagram. (c) Run of the density as a function of the temperature. (d) Differential emission measure for the solutions having a temperature inversion at the apex.

such gravity potentials, loops with temperature inversions at their apex do exist under certain conditions. In that case the pressure first drops and then starts to increase again towards the apex so that $E_{\text {heat }} / p^{2}$ first increases and then decreases again. In Fig. 7 we show the diagnostic diagram and the phase plane for seven values of $E_{\text {heat }}$. For high values of $E_{\text {heat }}$ only hot apex solutions exist (crossing of $F_{\mathrm{c}}=0$ on the right; case 1). Continuing the integration for these solutions in the upper half of the phase plane shows that the cool solutions run into the region where the coronal approximation breaks down. Decreasing the heating makes a class of solutions accessible which can have both hot or cool apexes (cases $2-5$ ). By further decreasing the heating the (cool) apex temperature shifts towards 
the maximum temperature in the loop until a heating rate is reached at which the cool and hot apex solutions coincide. This behaviour explains Fig. 1 of Collier Cameron (1988). For a further reduction of the heating no solutions at all can be found (cases 6 and 7) because the pressure starts to increase so rapidly, due to the reversed gravity, that the point where $F_{\mathrm{c}}=0$ cannot be reached. In Fig. $7 \mathrm{c}$ we show the run of the density with temperature for loops having a temperature inversion at their apex. These loops are characterized by a strong shoulder in the DEM (Fig. 7d) which can serve as a diagnostic tool. Intuitively one may argue that if the plasma in a loop experiences a reversed gravity (acceleration towards apex), that this would result in a strong increase of the density. This is in general not the case. Because $d \ln n / d s=d \ln p / d s-d \ln T / d s$, a strong increase of the density can be expected when $d \ln p / d s>d \ln T / d s$. For hot apex solutions one generally finds that the reverse holds and that the density continues to decrease despite that $d \ln p / d s>0$. Only for loops with temperature inversions, for which $d \ln T / d s<0$, a considerable increase of the density towards the apex is found (see Fig. 7c). The shoulder in the DEM indicates that strong emission can be expected from such loops. In VDOZ we show that in Algols and RS CVn-systems loops, penetrating the Roche lobe of the companion star near the $L_{1}$-point, can also have shoulders in the DEM due to the gravity reversal. This suggests that (relatively) strong emission can arise from the region in-between the stars. Note that if loops connecting both stars exist in these systems, these loops will not show exceptional emission because each half-loop can be considered as a normal loop.

\section{Conclusions}

In this review we have discussed various aspects of the modeling of quasistatic coronal loops. We demonstrated the use of the $\left(T, F_{\mathrm{c}}\right)$ phase plane for showing the behaviour of the solutions and showed that a diagnostic diagram, depicting $E_{\text {heat }} / p^{2}$ and $\Psi(T) /(2 k T)^{2}$, is useful to identify the critical points of the system and to determine the sign of $d F_{\mathrm{c}} / d s$ at a specific temperature. Our conclusions can be summarized as follows: 1) constant pressure loops or loops obeying hydrostatic equilibrium cannot have a temperature inversion near their apex because the apex condition $F_{\mathrm{c}}=0$ cannot be satisfied at $\left.T<T_{\mathrm{i}} ; 2\right)$ as a consequence the thermal instability predicted by Hood and Priest (1979) does not exist; 3 ) the boundary conditions $T_{\mathrm{b}}$ and $F_{\mathrm{c}, \mathrm{b}}$ must chosen in such a way that the corresponding phase plane trajectory connects to the chromosphere; 4) within the context of quasistatic coronal loops, spectroscopic studies do not provide information on the heating mechanism, despite numerous claims in observing proposals. Below $T_{\mathrm{i}}$ heating is unimportant while the range $T_{\mathrm{i}}<T \leq T_{\mathrm{a}}$ is almost 
isothermal; 5) heating must take place near the apex of loops, and the heating and radiative losses can only balance at one temperature in a loop. Esoteric heating functions do not exist; 6 ) the loop cross-section $A$ acts as an extra weighting function in the DEM so that by adjusting $A$ almost any observed DEM can be mimicked. This limits the diagnostic capabilities of DEM analyses; 7) loop expansion make the DEM distribution steeper while hydrostatic equilibrium makes it less steep; 8 ) for exponen ially expanding loops we presented new classes of solutions which predict strong emission from the apex of arcade structures, or the presence of cool material without the need of a thermal instability; 9) loops with a temperature inversion can only be found when there is a gravity reversal in the loop (fast rotating stars, in-between binary components). These loops have a strong shoulder in their DEM distribution indicating that strong emission can arise near the co-rotation radius of fast rotators or near the $L_{1}$ point in binaries. Interconnecting loops in binaries do not have a temperature inversion because the direction of the gravitational acceleration is 'downward' in each of the loop-halves.

\section{References}

Bray, R.J., Cram, L.E., Durrant, C.J., Loughhead, R.E. (1991) Plasma loops in the solar corona. Cambridge Astroph. Ser., Vol. no. 18, Cambridge University Press

Clark, B.G., Kellermann, K.I., Shaffer, D.B. (1975) ApJ, 198, L123

Clark, T.A. et al. (1976) $A p J$, 206, L107

Collier Cameron, A. (1988) MNRAS, 233, 235

Craig, I.J.D., McClymont, A.N., Underwood, J.H. (1978) $A \& A, 70,1$

Hood, A.W., Priest, E.R. (1979) $A \& A, 77,233$

Hood, A.W., Anzer, U. (1988) Solar Phys., 115, 61

Kuin, N.P.M., Martens, P.C.H., (1982) $A \& A$, 108, L1

Landini, M., Monsignori Fossi,B.C. (1981) $A \mathcal{E} A$, 102,391

Lestrade, J.F., et al. (1984) $A p J, 279,184$

Mutel, R.L., Doiron, D.J., Lestrade, J.F., Phillips, R.B. (1984) ApJ, 278, 220

Preś, P., Siarkowski, M., Sylwester, J. (1995) MNRAS, 275, 43

Rosner, R., Tucker, W.H., Vaiana, G.S. (1978) ApJ, 220, 643 (RTV)

Schrijver, C.J., (1989) ApJ, 341, 484

Serio, S., Peres, G., Vaiana, G.S., Golub, L., Rosner, R. (1981) ApJ, 243, 288

Siarkowski, M (1992) MNRAS, 259, 453

Spitzer, L. (1962) Physics of fully ionized gasses. Interscience, New York

Stern, R.A., Uchida, Y., Tsuneta, S., Nagase, F. (1992) ApJ, 400, 321

van den Oord, G.H.J., Barstow, M.A. (1988) $A \& A$, 207, 89

van den Oord, G.H.J., Mewe, R. (1989) $A \& A$, 213, 245

van den Oord, G.H.J., Zuccarello, F. (1996) $A \& A$, to be submitted (VDOZ)

Vesecky, J.F., Antiochos, S.K., Underwood, J.H. (1979) $A p J, 233,987$ (VAU)

Walter, F.M., Gibson, D.M., Basri, G.S. (1983) $A p J$, 267, 665

White, N.E., et al. (1986) ApJ, 301, 262

White, N.E., Parmar, A.N., Sweeney, M.A., Culhane, J.L. (1987) MNRAS, 227, 545

White, N.E., Shafer, R.A., Horne, K., Parmar, A.N., Culhane, J.L. (1990) ApJ, 350, 776 


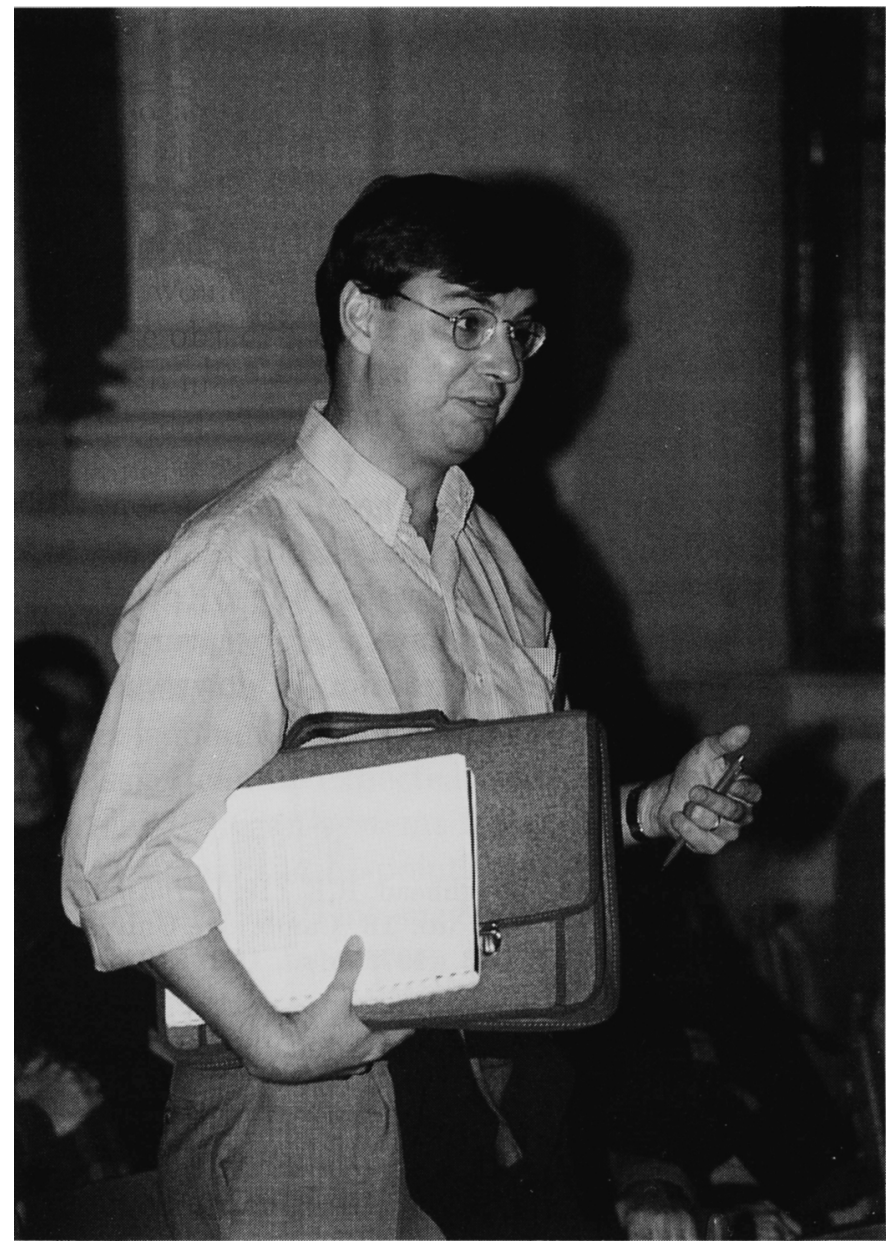

Bert van den Oord at one of the most important tasks of a scientific meeting: asking nasty questions. (He is good in that!). 